\title{
Die Mikroskopie des lebenden Augenhintergrundes im fokalen Lichte der Gullstrandschen Nernstspaltlampe.
}

\author{
3. Mitteilung.
}

\section{Die pathologische Histologie der lebenden Netzhaut bei der Embolie der Zentralarterie unter besonderer Berücksichtigung der Streitirage einer intravitalen Gelbfärbung der Macula.}

Von

Dr. med. Leonhard Koeppe,

Privatdozent für Augenheilkunde an der Universitäts-Augenklinik zu Halle a. $\mathrm{s}$.

Mit Tafel II.

\section{Inhalt.}

A. Einleitung; speziellere technische Bemerkungen.

B. Das Spaltlampenbild der lebenden Netzhaut bei der Embolie der Zentralarterie:

a) im Bereiche der Macula; die Frage der Gelbfärbung derselben;

b) im Bereiche der übrigen Netzhaut;

c) im Bereiche der Papille sowie deren engerer Umgebung.

C. Zusammenfassung und Schlußergebnisse.

D. Literatur.

Die Mikroskopie des lebenden Augenhintergrundes an der Nernstspaltlampe mit Hilfe des in der ersten Mitteilung beschriebenen Silberspiegels sowie des Auflageglases auf die lebende Hornhaut bei starker Vergrößerung läßt uns, wie in der zweiten Mitteilung gezeigt wurde, nicht nur in der lebenden normalen Netzhaut eine Fülle neuer und interessanter Beobachtungen anstellen, sondern gerade das Gebiet der pathologischen Augenhintergrundsveränderungen eröffnet unserer Forschung ein dankbares und auch bedeutsames Arbeitsfeld. In den folgenden Mitteilungen sollen num des weiteren die der neuen Untersuchungsmethode zugänglichen Krankheitsbilder eingehend und methodisch besprochen und die histologischen Veränderungen geschildert werden, welche uns das Spaltlampenbild dabei darbietet.

Wir beginnen die Besprechung der Augenhintergrundspathologie im fokalen Lichte der Nernstspaltlampe mit der Darstellung der Netzhautveränderungen bei der Embolie der Zentralarterie. Veranlassung 
daza gab uns einmal die Beobachtung zweier ${ }^{1}$ ) sehr schöner und instruktiver Fälle mit unserer neuen Untersuchungsmethode, andererseits aber ein Problem, das zwar zum Teil hoch in das Gebiet des Physiologischen gehört, andererseits aber mit der Netzhautpathologie bei der Embolie der Zentralarterie so innig und untrennbar zusammenhängt, daß es nur gelegentlich der Schilderung dieses Krankheitsbildes besprochen werden kann, wie im folgenden gezeigt werden wird.

Einige speziellere technische Bemerkungen über die Untersuchungsmethodik und -apparatur seien an dieser Stelle noch eingeschaltet.

Einmal benutzten wir für den ersten beobachteten Fall noch den Nernstkörper als Lichtquelle der Spaltlampe, doch haben wir unsere Hauptstudien mit der spezifisch helleren Lichtquelle des Nitralichtes an dem zweiten Falle vorgenommen. Auf die nähere Einrichtung und Anwendung dieses neuen Lichtes hatte ich bereits in der vorhergehenden Arbeit schon hingewiesen. Zweitens verwendeten wir bei der Untersuchung des zweiten Falles den ebenfalls schon daselbst geschilderten Blendenaufsatz resp. die Schlitzverengerung des Diaphragmas an der asphärischen Beleuchtungslinse. Mit diesen Verbesserungen ausgerüstet konnten wir die Beobachtungsergebnisse des ersten Falles an dem zweiten desto schöner bestätigt finden.

Für die Beobachtungen verwendeten wir sowobl den direkten als auch den indirekten Beleuchtungs- resp. Untersuchungsmodus. Namentlich die indirekte Beleuchtung erwies sich für bestimmtere Feststellungen als wertvoll, worauf ich gelegentlich der Schilderung meiner Befunde noch genauer zu sprechen kommen werde.

Der Umstand, daß bei unseren beiden Fällen die Augenmedien völlig klar erschienen und auch keine Refraktionsanomalien bestanden, erleichterte uns die Untersuchung mindestens ebenso wie das jugendliche Alter der Patienten zwischen 30 und 35 Jahren.

Das oben schon angedentete Problem, welches mit der Behandlung des Spaltlampenbildes der Embolie der Zentralarterie aufs innigste verknupft erscheint, ist, wie auch die Durchsicht der Literatur uns noch zeigen wird, die alte, noch ungelöste Streitfrage, ob der Bezirk des schärfsten Sehens in der lebenden Netzhaut, die Macula centralis, tatsächlich gelb gefärbt ist oder nicht. Hier war unserer Forschung an der Nernstspaltlampe auf diese Weise ein besonderes Arbeitsgebiet erschlossen. Ich beginne daher unsere heutige Schilderung zunächst mit der Erörterung und kritischen Beleuchtung der Frage einer intravitalen Gelbfärbung der Macula und werde daran anschließend die im Fokus

1) Ein dritter Fall, der eine 30 jährige Frau betraf, zeigte genau die gleichen und im folgenden beschriebenen Spaltlampenbilder des Augenhintergrundes, kam aber erst $\mathrm{nach}$ Drucklegung der Arbeit zur Beobachtung. 
der Spaltlampe sichtbaren weiteren Netzhaut- resp. Opticusveränderungen zur Diskussion zu stellen haben.

Bekanntlich war ja bis jetzt die Entscheidung nicht möglich gewesen, ob die Macula im Leben wirklich eine gelbe Farbe besitzt, und zahlreiche Autoren haben die Lösung dieses Theorems gesucht, ohne jedoch bisher einen unbestrittenen Beweis ihrer für eine Gelbfärbung der Macula spreehenden Befunde erbringen zu können.

Wohl nahm bisher die weitaus größere Hälfte der Autoren an, daß im toten Auge ein diffuser gelber, in Alkohol löslicher Farbstoff den eigentlichen Bezirk der Macula sowie auch deren zentrale Fovea durchtränke, doch konnte eben bis heute dieser Farbstoff in der lebenden Retinamitte noch nicht einwandfrei festgestellt werden.

Lange wogte so der Widerstreit der Ansichten bei den verschiedenen Autoren hin und her, ohne jedoch bislang einen bestimmteren und allgemeingültigeren Ausklang gefunden zu haben.

So erinnere ich nur kurz an die Beobachtungen von Dimmer, der bei der gewöhnlichen ophthalmoskopischen Untersuchung unter Benutzung sehr hellen Tageslichtes in einigen stark pigmentierten kindlichen Augen eine deutlich gelblichere Färbung. des Netzhautzentrums wahrgenommen haben will, eine Erscheinung, die er auch in einigen Fällen von Blutmangel der Netzhaut infolge einer Verstopfung der Zentralgefäße des. Sehnerven angedeutet sah.

Dieser Auffassung Dimmers, daß es sich bei der von ihm in den angeführten Fällen gesehenen gelben Färbung der Maculagegend tatsächlich um eine Eigenfarbe der Netzhaut an dieser Stclle handeln solle, vermochte sich Gullstrand nicht anzuschließen. Gullstrand widerlegte die Deutung Dimmers und konnte zeigen, daß bei zunehmender Intensität des auf die Netzhaut auffallenden Lichtes im Grunde der Fovea centralis die Farbe des Epithelpigmentes bzw. bei sparsameren Pigmenten die Mischfarbe dieser Farbe mit der Blutfarbe des Augenhintergrundes gesehen wird.

Vor allem bei der durch Gefäßverstopfung erzeugten Netzhautischämie, woselbst nach diesen Deduktionen Gullstrands doch die gelbe Farbe des Netzhautzentrums gewissermaßen als Deckfarbe hervortreten müßte, weil hier durch die weiße Transsudation in die Netzhaut das in der unmittelbaren Nachbarschaft der Fovea vom übrigen Augenhintergrunde reflektierte Licht aufgehoben ist, vermißte Gullstrand die fragliche gelbe Eigenfärbung der Netzhaut im Bereiche der Macula.

Dagegen erwähnte nun wieder J. van der Hoeve einen Fall, bei dem dureh einen Kuhhornstoß, der einen 16 jährigen Patienten getroffen hatte, die Arteria centralis retinae resp. der ganze Sehnerv zerrissen war. Bei engen Arterien und normalen Venen fand sich das 
typische Bild der Verstopfung der Zentralarterie im Sehnerven, eine dichte milchigweiße Trübung der Netzhaut um die Stelle des schärfsten Sehens herum. Ein noch von annähernd normaler Augenhintergrundsfarbe erscheinender Fleck schläfenwärts vom Orte des Sehnerven zeigte sich funktionsuntüchtig, woraus geschlossen wurde, daß der,ganze Sehnerv ebenfalls zerrissen war, weil bei der einfachen Verstopfung der Sehnervenzentralarterie diese Netzhautpartien infolge besonderer Blutversorgung aus dem Sehnerven - mittels der sogenannten cilioretinalen Arterien - funktionstüchtig zu bleiben pflegen.

Die gesamte Macula erschien hier in einer Ausdehnung von etwa 1/2 Pupillendurchmesser gelb mit etwas dunklerem Zentrum. Da die sonst in der Fovea centralis bei der Embolie der Zentralarterie erscheinende rote Stelle, welche gewissermaßen wie durch ein Fenster in der dünnsten Netzhautstelle hier die rote Augenhintergrundsfarbe durchscheinen läßt, zu, vermissen blieb, so erklärte sich v. d. Hoeve dieses Verhalten damit, daß in diesem Falle durch Zerreißung des Sehnerven auch der hinter der getrübten Netzhaut gelegene Augenhintergrund anämisch war, so daß die gelbe Eigenfarbe des Netzhautzentrums zur Geltung kommen konnte.

Bemerkt sei noch, daß der erwähnte Befund der Kontrolle und des Vergleiches halber von v. d. Hoeve sowohl bei Auerlicht als bei elektrischem Licht und Tageslicht ophthalmoskopisch erhoben and bei jeder Beleuchtungsart in gleicher Weise bestätigt werden konnte.

Einen ährilichen Ophthalmoskopiebefund im rothaltigen Lichte, wie Dimmer und v.d.Hoeve, erhielt auch Vogt') in einem Falle, doch verschwand allmählich die gelbe Maculafarbe und war dann auch im rotfreien Lichte nicht mehr sichtbar.

Während nun Gullstrand in dem nur minimale Spuren von Rot enthaltenden Linienspektrum der Quecksilberdampfbogenlampe in der gesamten Maculagegend des Auges keinen gelberen Farbenton erkennen konnte, als ein ganz dunkler Augengrund bei diesem Lichte zu zeigen pflegte, rückte die ganze Frage in ein neues bedeutsames Stadium, als es Vogt gelungen war, durch Vorschaltung eines KupfersulfatEreoviridinfilters aus dem Lichte einer Ophthalmoskopierbogenlampe alles Rot und einen Teil des Orange zu entfernen. In diesem Lichte sah er die Macula in toto auch in völlig normalen Auge stets leuchtend gelb gefärbt. Vogt erklärt dieses Phänomen damit, daß die gelbe Farbe der Netzhaut in deren dünneren und dünnsten Partien als Lackfarbe erscheint, die durch die Beleuchtung des von der Sclera, der Aderhaut und dem Pigmentepithel her reflektierten rotfreien Lichtes vom Beobachter so gesehen wird, wie etwa ein auf rotem Papier liegendes Gelbglas in seiner Eigenfarbe hervortritt, wenn das rotfreie Licht mit ge-

\footnotetext{
1) A. Vogt, Zur Farbe der Macula retinae. Klin. Mon. f. A. 60. S. 450. 1918.
} 
nügender Intensität auffällt und von dem jetzt fast schwarz aussehenden roten Papiere in immer noch hinreichender Stärke reflektiert wird. In der übrigen Retina wird dagegen das rotfreie Licht so stark diffus reflektiert und die Retina für dieses Licht so undurchsichtig gemacht, daß sie blaugrün erscheint, während das eigentliche Rot oder Rotgelb des Pigmentepithels resp. der Aderhaut sowie auch das an der Sclera reflektierte Licht völlig verdeckt wird.

Vogt sah nun die gelbe Lackfarbe der Macula nur dann, wenn die Lichtstärke des rotfreien Lichtes eine hinreichende war. Er vertritt somit den Standpunkt, daß das Licht von den tieferen Teilen reflektiert werden müsse, um die besagte Lackfarbe der Macula erkennen zu können. Bei herabgesetzter Intensität des rotfreien lichtes verschwand relativ frühzeitig die gelbe Farbe der Macula und machte einer mehr oder minder deutlichen braunroten Färbung Platz. Hier verlosch also die gelbe Farbe der Netzhaut in dem Momente, wo das von den unter der dünnsten Netzhautstelle reflektierte Licht unter die Wahrnehmungsschwelle des Beobachters trat, andererseits sah Vogt die gelbe Farbe eben nur dann, wenn das im Netzhautzentrum aus den tieferen Teilen reflektierte Licht nach Durchgang durch die Netzhaut über dieser Schwelle lag.

Die Folgerung Vogts, daß der gesamte, im rotfreien Lichte gelb gesehene Netzhautbezirk die gelbe Eigenfarbe der Macula darstelle und eben aus der Lackfarbe der Netzhautmacula stamme, läBt nun Gullstrand nicht gelten und bestreitet die Gelbfärbung.

Nach Gullstrand muß das von den tieferen Teilen reflektierte Licht in der Mitte der Fovea, woselbst die Netzhaut am dünnsten ist, bei hoher Beleuchtungsintensität mehr oder minder gelb hervortreten. Bei Vorhandensein einer gelben Lackfarbe in der Netzhaut könnte daher diese gelbe Farbe bei intensivem Vogtschen Lichte nicht an der dünnsten Netzhautstelle gesehen werden. Liegt dagegen das von den tieferen Teilen reflektierte Licht unter der Schwclle der Wahraehmung, so zeigt die Macula im rotfreien Lichte keine gelbe Farbe, woraus nach Gullstrand geschlossen werden kann, daß bei der Vogtschen Untersuchungsanordnung die Netzhautmitte unmöglich eine gelbe Eigenfarbe besitzen dürfte. Nach der Erklärung Gullstrands sieht man mithin im rotfreien Lichte von Vogt die gelbe Eigenfarbe der tieferen Augenhintergrundsteile durch die dünnste Netzhautstelle gewissermaßen wie durch ein Fenster hervorleuchten, während bei sinkender Beleuchtungstärke dieses Hervorleuchten erlischt und die Eigenfarbe des Pigmentepithels der Netzhautmitte als mehr oder minder dunkelbraunrote Tönung hervortritt.

Aus all dem Gesagten und bisher über den vorliegenden Gegenstand bekannt Gewordenen geht nun offenkundig hervor, daß es bis jetzt 
nicht gelungen ist, die Existenz eines gelben Farbstoffes im Bezirke des schärfsten Sehens in der lebenden Netzhaut einwandfrei nachzuweisen und damit annähernd eindeutig die Streitfrage zu entscheiden, ob die lebende Macula centralis der Netzhaut gelb gefärbt ist oder nicht. Es erscheint daher um so verständlicher, daß wir uns mit der Lösung des Problems der Stereomikroskopie des lebenden Avgenhintergrundes im fokalen "Lichte der Spaltlampe vermittels des auf die Hornhaut des untersuchten Auges aufgesetzten Kontaktglases vor allem dieser Frage zuwandten und danach trachteten, die lebende Macula auf ihre Farbe im natürlichen, also rothaltigen und den Augenhintergrund durch eine bestimmtere Eigenfarbe nicht becinflussenden Lichte zu prüfen.

Die strengste Beachtung dreier Untersuchungsbedingungen war uns dabei von vornherein klar.

Einmal war es die Unmöglichkeit, die lebende Netzhautmitte auf ihre im natürlichen Lichte der Spaltlampe vorhandene Eigenfarbe zu untersuchen, solange die Netzhaut"von normaler Durchsichtigkeit, also der Augenhintergrund überhaupt normal war. Hier hätten wir auch im engstumschriebenen fokalen Lichtkegel unserer Nernstspaltlampe infolge der Durchsichtigkeit und Dünnheit der Netzhaut, speziell in deren Macula, immer nur die Farbe des von dem Pigmentepithel, der Aderhaut und Sclera nach zweimaligem Durchgange durch die ersteren beiden reflektierten, relativ langwelligen Lichtes, niemals aber eine Eigenfarbe der betreffenden davorliegenden Netzhaut zu sehen bekommen, also stets nur das bekannte mehr oder minder dunkle Braunrot der Macula. Auch im von jeder Spur von Rot befreiten Lichte der Spaltlampe, was durch Vorschaltung eines Vogtschen Filters vor den Spalt der Lampe ja unschwer zu erreichen ist und ich an anderer Stelle zeigen konnte, hätten wir die Frage einer dort existierenden Eigenfarbe der Netzhaut nicht unbestritten lösen können, wie die oben angeführten Gegengründe Gullstrands grgen Dimmer und gegen die Vogtsche Deutung der im rotfreien Lichte deutlich gelb erseheinenden Macula beweisen.

Aus allen diesen Gründen mußten wir also darauf verzichten, in der normalen und völlig durchsichtigen Netzhautmacula den vielumstrittenen gelben Farbstoff zu suchen. Hier konnte nur eine Netzhauttrübung zur Untersúchung verwendet werden, die sich ausschließlich in den vorderen Netzhautschichten abspielte - und das ist, wie auch die noch umstrittenen und noch nicht einwandfreien Beobachtungen von v. d. Hoeve und Lottrup-Andersen, der eine der Beobachtung v. d. Hoeves ähnliche Erscheinung bei Verstopfung der Zentralarterie der Netzhaut sah, beweisen, eben dieses letztere Krankheitsbild, aber in der richtigen Deutung der an der Spaltlampe ganz anderen und die Fehlerquellen 
der genannten Autoren ausschließenden Untersuchungsbedingungen.

Und diese richtigen Untersuchungsbedingungen, welche uns für eine mit allergrößter Wahrscheinlichkeit richtige Deutung des Gesehenen die beste Gewähr bieten, gibt uns einzig und allein der exakt dirigierbare und nur eine äußerst kleine und umschriebene Gewebsstelle auf dem lebenden Augenhintergrunde hell beleuchtende fokale Lichtkegel der Nernstspaltlampe an die Hand und läßt uns unter folgenden für die Entscheidung der Frage fundamental wichtigen und neuen, bisher nicht anwendbar gewesenen Versuchsbedingungen arbeiten.

Die eine dieser Bedingungen ist die Möglichkeit, unter völliger Dunkelheit des bei der Verstopf ung der Zentralarterie des Sehnerven kirschrot hervortretenden eigentlichen Foveateiles der Macula, also unter mehr oder weniger völligem A usschlusse des durch dieses Fenstervonden tieferen Teilen des Augengrundes kommenden Lichtes systematisch allein die der Fovea unmittelbar benachbarten vorderen Netzhautschichten im natürlichen Fokallichte der Spaltlampe a uf ihre Eigenfarbe zu untersuchen. Denn, wie wir oben schon begründeten, ist über der eigentlichen Fovea die Netzhaut so dünn, daß bei der infolge der Embolie stattfindenden weißlichen Trübung der vorderen Netzhautschichten diese Stelle von der Trübung verschont bleibt, zumal auch die in dieser dünnen Stelle gelegenen Netzhautschichten durchweg den von der Veränderung fast unberührt bleibenden tieferen Lagen dieses Gewebes angehören. Hier lag, wie Gullstrand angibt, der eine Fehler der bisherigen Untersuchungen. Denn hier kann leicht das durch das ,Foveafenster" von den tieferen Augengrundsteilen reflektierte Ticht in der Fovea wie auch in der engeren Umgebung der die Fovea begrenzenden Maculapartien die gelbe Farbe vortäuschen, wenn, wie das in den bisherigen Untersuchungsmethoden üblich war, mit mehr oder weniger zerstreutem Lichte beobachtet wurde.

An der Spaltlampe kann dagegen dieser Fehler durch schärfste Konzentration des Fokus auf die einzelnen Zonen außerhalb des „Fensters $^{6 \text { v }}$ vermieden und die getrübte Gewebslage der Netzhaut auf ihre Farbe untersucht werden. Hier wird also das gefährliche Fenster, der kirschrote Fleck, völlig ausgeschaltet und ohne die Gefahr eines von hinten her reflektierten und von dort durchscheinenden gelbroten resp. annähernd gelben Lichtes untersucht.

Ein anderer ausschlaggebender Vorteil der Spaltlampenuntersuchung der vorliegenden Streitfrage besteht in der Möglichkeit, die eventuell gefärbte Zone a uch im indirekten Lichte, d. h. mehr oder weniger im D unkelfelde, zu untersuchen. Dies geschieht, wie wir schon öfters sahen, ja einfach dadurch, daß man den beleuchteten Punkt 
in der eingestellten und fraglichen gefärbten Partie neben den unmittelbar beobachteten Punkt verlegt; man kann dann auch die Ausdehnung der gefärbten Partie nach hinten zu einigermaBen abschätzen.

Der dritte und letzte Vorteil betrifft die starke Vergrößerungsmöglichkeit der betreffenden Gewebsstelle bis zu etwa 70 fach linear; mit dieser Vergrößerung hatte im fokal beleuchteten Spaltlampenbilde noch niemand die Macula im natürlichen Lichte auf ihre Naturfarbe hin untersucht. Bisher war die für diese Untersuchung angewendete Vergrößerung noch nicht über $16 \mathrm{fach}$ zu steigern gewesen, abgesehen vom Gullstrandschen Ophthalmoskop, das uns aber wegen der zu diffusen und zu schwachen Beleuchtung der Macula in der vorliegenden Streitfrage einer intravitalen Gelbfärbung der Macula auch nicht weiterzubringen vermochte. Dieses muß auch für die Untersuchungsmethode der reflexlosen Mikroophthalmoskopie von H. Wolff gelten, denn bei dieser Methode ist trotz stärkerer Vergrößerung das Licht wiederum. zu gelblich und vor allen Dingen auch in seiner Intensität zu schwach.

So darf ich denn nunmehr auf die schon oben kurz angedenteten beiden selbst beobachteten Fälle zu sprechen kommen.

Es fanden sich klinisch wie vor allem auch ophthalmoskopisch in beiden Fällen genau die gleichen Bilder, beide Fälle kamen am zweiten Tage nach der Embolie zur Beobachtung und zeigten ophthalmoskopisch die bekannte ausgedehnte weißliche Trübung der vorderen Netzhautschichten unter Aussparung der kirschrot als „Fenster" durchschimmernden zentralsten Foveapartie, während eine länglich polygonale cilioretinal versorgte Netzhautpartie temporal der Papille normal erschien und sich noch nicht ganz bis an die äußerste Peripherie der Maculagegend heran erstreckte.

Durch die völlig klaren und außer einigen bei der gewöhnlichen Spaltlampenuntersuchung sichtbaren roten und weißen Blutzellen keine weiteren Besonderheiten zeigenden Augenmedien sah man mit unserer Apparatur - vor allem bei der 32 jährigen Helene W. - die im ophthalmoskopischen Bilde kirschrot erscheinende zentrale Foveapartie ebenfalls in dieser Farbe mit deutlicher schöner Körnelung des Pigmentepithels, wie sie auch auf der beigegebenen Abbildung zum Ausdruck kommt.

Konzentrierte man nun völlig außerhalb dieser Partie das Spaltbüschel - vor allem bei Ausdehnungsbeschränkung des letzteren vermittels der erwähnten Diaphragmaverengerung - Zone um Zone auf die aufsteigenden Partien der Fovea resp. auf den dieselbe umgebenden Ringwall der Macula, so sah man sowohl im"direkt wie auch im indirekt beleuchteten Bilde mit wundervoller Deutlichkeit und Plastik in den von der schneeweißen Trübung ergriffenen vorderen Netzhautpartien, also bei völliger Dunkelheit des kirschroten Fensters sowie a uch von dessen unmittelbarer Umgebung, eine aller- 
feinst chagriniert hervortretende prachtvoll goldgelbe bis goldgrüne Ockerfarbe, die in der Umgebung der roten Fovea, welche von horizontal leichtelliptischer Gestalt und um vieles tiefer liegend gegenüber den stark aufgequollenen übrigen Maculapartien trichterförmig eingesenkt sich darstellte, am intensivsten erschien und sich dann ganz allmählich nach dem genannten Ringwalle zu verlor $\boldsymbol{x}$ ). Dabei betrug der Durchmesser der gefärbten Partie etwa $1 / 2$ Papillendurchmesser.

Wie auch die beigegebene Abbildung lehrt, fand sich des weiteren in der Peripherie der eigentlichen Foveaeinsenkung ein ebenfalls horizontal-elliptisch verlaufender dunkler Schatten, in dessen Bereiche von der roten Farbe der Fovea kaum etwas erkenntlich war und der allmählich in die genannte Farbe der Foveatiefe überging.

Während nun die weitere Umgebung der gelb gefärbten Partie bei der ersten Untersuchung, also am 2. Tage nach der Embolie, an der Spaltlampe einige weiter unten zu besprechende Besonderheiten bot, war auch ophthalmoskopisch bei der 16 fachen Vergrößerung des aufrechten Bildes von der gelben Farbe ebensowenig zu erkennen wie bei ca. 40facher Vergrößerung im Bilde des Gullstrandschen Ophthalmoskops.

Auch bei herabgesetzter Beleuchtungsstärke des fokal beleuchteten Spaltlampenbildes durch Spaltverengerung oder Vorschaltung rauchgrauer Gläser blieb die gelbe perifoveale Maculafarbe immer noch sichtbar, obwohl schließlich die rote Farbe der eigentlichen zentralen Fovea verschwand, also trotz unter der Erkennbarkeitsschwelle des vom fovealen Pigmentepithel diffus reflektierten Lichtes. Schon dadurch dürfte nunmehr der Beweis erbracht sein, da $B$ die vorderen Retinaschichten $z u m$ mindesten in der Umgebung der eigentlichen Fovea centralis bis etwa zum Beginne des Ringwalles im lebenden. A uge eine gelbe Farbe besitzen ${ }^{2}$ ) und die Forderung eindeutig erfüllen, welche Gullstrand für den Nachweis einer gelben Farbe in dieser Gegend des lebenden Auges a ufstellte.

Damit ist aber auch der Beweis geliefert, daB die Behauptungen Vogts betreffs der Existenz einer gelben

1) Bei unserem eingangs nachträglich zitierten dritten Falle von Embolie war die Gelbfärbung, da bei der exsten Untersuchung - drei Wochen nach Eintritt der Embolie - bereits eine weitgehende Aufhellung des Augenhintergrundes wieder eingetreten war, nur noch in der temporalen Umgebung der Fovea zu erkennen. Im übrigen unterschieden sich die in den weiteren Stadien des Verlaufes beobachteten Spaltlampenbilder in nichts von den Bildern bei den geschilderten beiden Fällen.

$\left.{ }^{2}\right)$ Vgl. die vorläufige Mitteilung über den Nachweis der Gelbfärbung in der Münch. med. Wochenschr. Nr. 43, 1918.

Daß bei dem Zustandekommen der gelben Farbe das Pigmentepithel, wie Vogt schon annahm, nicht beteiligt sein kann, geht unseres Erachtens nach gleichfalls daraus hervor. 
Lackfarbe in der lebenden Macularetinae zu Recht bestehen und die von diesem Autor in seinerletzten Arbeit über den Gegenstand (Klin. Mon.f.A. Bd. 60. 1918) angeführten Kriterien - z. B. der Retinitis in macula mit weißen Plaques, die im rotfreien Lichte eine gegen die Umgebung hervortretende leuchtend gelbe Farbe zeigten und damit als Deckfarbe gelten konnten - den Tatsachen entsprechen und die Streitfrage der Gelbfärbung der lebenden Macula entsebeiden.

Die Befunde Vogts würden auch dadurch unwiderlegliche Beweis kraft gewinnen, wenn seine Befunde im rotfreien Lichte an weiteren Fällien von Embolie der Zentralarterie nachgeprüft würden und festgestellt werden könnte, wie sich hier bei der dichten weißlichen Netzhauttrübung unter Ausschluß des von den tieferen Augenteilen zurückreflektierten Lichtes die Farbe der Fovea und speziell der perifovealen Maculapartien verhielte. Wir selber haben zwar diesen Versuch im rotfreien Lichte der Spaltlampe unter Vorschaltung des Vog tsehen Filters ebenfalls angestellt, konnten jedoch wegen der durch das Filter bedingten Intensitätsschwächung des fokalen Lichtbüschels noch zu keinem eindeutigeren Resultate gelangen. Da von den Zeißwerken eine weitere Intensitätssteigerung der Spaltlampenlichtquelle, wie ich hörte, versucht werden soll, so werden wir späterhin an weiteren Fällen von Embolie diese Untersuchungen durchzuführen haben.

Der Einwand, daß auch die Farbe unseres natürlichen Spaltlampenlichtes die gelbe Farbe wenigstens zum Teil mitbedingen konnte, ist deshalb nicht stichhaltig, weil auch die Vorschaltung verschieden getönter Gelb- und Blauscheiben vor den Spalt sowohl bei der Nernstlichtuntersuchung des ersten Falles wie auch bei der Nitraliohtbeleuchtung des zweiten Falles an dem erhaltenen Resultate nichts änderte. Speziell dureh die schwache Gelbscheibe wurde ja das an kurzwelligeren Strahlen relativ reichlichere Licht der Nitralampe dem Tageslichte annähernd gleich gestaltet.

Auch der Einwurf, daß bei unserer Untersuchung im natürlichen Lichte das von hinten her neben der Fovea durchscheinende rote Licht der tieferen Hintergrundsteile die Farbe bedingen könne, ist deshalb hinfällig, weil dann das perifoveale, von uns gesehene Maculagelb um vieles rötlicher auf dem weißgetrübten Untergrunde der vorderen Retinaschichten erscheinen müßte, was eben durchaus nicht der Fall ist.

Wenn auch nach unseren Befunden eine im Lében vorhandene Gelbfärbung der rot erscheinenden zentralen Foveapartien noch nicht nachgewiesen werden konnte, weil bei unseren Fällen die Fovea selbst infolge ihrer mangelnden weißen Trübung rot erseheinen mußte, so bleibt allerdings die endgültige Entscheidung der Frage einer intravitalen Gelbfärbung der Fovea bis auf weiteres noch ungelöst, wenn 
auch die Annahme einer solchen Färbung in Anbetracht der von uns wohl einwandfrei bestätigten intravitalen Gelbfärbung der übrigen Maculapartien zum mindesten recht wahrscheinlich gemacht worden ist.

Vielleicht gelingt es hier noch einmal, bèi Netzhauttrübungen, die auch den Grund der Fovea centralis in die weißliche Undurchsichtigkeit miteinbeziehẻn, ebenfálls einen intravital existierenden gelben Farbstoff im Netzhautinneren nachzuweisen. Allerdings bliebe hier stets zu berücksichtigen, daß die weißliche Trübung der Retina am Grunde der Fovea nur in so dünner Schicht vorhanden wäre, daß die Gefahr bestünde, einen Teil wenigstens der gesehenen gelben Farbe auf Rechnung des von den tieferen Teilen her reflekțierten Lichtes zu erhalten und dem von der getrübten Netzhautfovea zurückgestrahlten Lichte der fraglichen gelben Farbe beigemischt zu sehen, was für die um vieles dickeren und dichter getrübten perifovealen Maculapartien bei ausschließlicher fokaler Beleuchtung dieser sicher auszuschließen war.

Wie die genauere mikroskopische Untersuchung der nicht gelb gefärbten und weiß getrübten vorderen Netzhautpartien an der Spaltlampe ergab, kamen im Bereiche der den gelbgefärbten Teilen unmittelbar benachbarten Gewebszonen in der Netzhaut befindliche Blutungen feinster Art oder deren Umwandlungsprodukte nicht in Betracht, so daß damit der Einwand hinfällig wird, es könne sich in meinen Fällen bei der Gelbfärbung vielleicht um solche hämatogene Farbstoffe gehandelt haben. Das gilt auch für die Annahme eines Einflusses von ausgetretener Blutflüssigkeit, d. h. eines Ödems, wie es bei der embolischen Netzhauttrübung von Leber, Rubert, Siegrist u. a. festgestellt und für die weiße Farbe der Netzhaut bei diesem Krankheitsbilde verantwortlich gemacht wurde. Auch für die anatomisch bei der Embolie in den vorderen Netzhautschichten festgestellten zelligen Elemente, wie die von Leber angeführten Fettkörnchenzellen und Ähnliches, hätte diese Annahme Gültigkeit. Wären alle diese Gebilde von irgendwelcher Bedeutung für die Gelbfärbung, so hätte man auch außerhalb der Gelbfärbung in der Retina eine mehr oder minder angedeutete Gelbfärbung nachweisen müssen, was aber nicht der Fall war. Auch die Papille erschien, wie hiex schon vorausbemerkt sei, in ihrer an der Spaltlampe auch sonst sichtbaren rötlichen bis weißrötlichen Farbe in keiner Weise auffallend verändert, trotz der an ihr noch zu besprechenden embolischen Veränderungen.

Unter unseren Augen verlor sich nun bei dem ersten Falle von Tag zu Tag mit der Aufhellung der weißen Trübungszone infolge zum größten Teile sich spontan wiederherstellender Zirkulation die gelbe Farbe unter Wiederrötlichfärbung des Augenhintergrundes mehr und mehr. Dabei traten zuerst von den freigebliebenen normalen Netzhautpartien, die cilioretinal versorgt ersohienen, zungenförmige rote "Halbinseln" in 
Erscheinung, mit deren Ausbreitung auch die gelbe Farbe scheinbar immer unregelmäBiger und zerrissener wurde, während die vorderen Netzhautschichten daselbst ein entschieden körnigeres Aussehen gewamnen. Auch auf der temporalen Seite begann dann nach etwa $10 \mathrm{Ta}-$ gen das allmähliche Verschwinden der Farbe unter sonst gleichen Erscheinungen. Die Farbe, die zuerst ein schönes Ocker-bis Gold-oder auch Grüngelb darstellte, ging bei diesem Prozesse der langsamen Auflösung vor dem endgültigen Verschwinden erst in ein mehr schmutziges Gelbgrün, dann in ein Braungrün und schließlich in das Braunrot des gewöhnlichen Aussehens dieser Gegend an der Nernstspaltlampe über. Dabei flossen die ,Halbinseln“" zu größeren Flächen zusammen, bis schließlich mehr oder minder die ganze Macula ohne die gelbe Farbe in dem bekannten Braunrot ersehien, was auch ophthalmoskopisch zum Ausdruck kam.

Unter Abflachung der trichterförmig eingesenkten Fovea verschwand allmählich auch der geschilderte und auf der Abbildung sichtbare ringförmige Schatten in der Foveatiefenperipherie. Die rote Farbe der Fovea ging dann wieder mehr allmählich in die mehr braune Tönung der übrigen Macula über, wobei auch die Vorwölbung der letzteren verschwand und ungefähr die alte Konfiguration wieder annahm.

Während des Verlaufes der Aufhellung traten des weiteren sowohl unmittelbar in den vorderen Netzhautschichten der Fovea wie ganz besonders der Mácula und auch in den übrigen ursprünglich nach der Embolie weißgefärbten vorderen Netzhautschichten sehr deutlich allenthalben kleinste und zahlreiche Cholesterinkrystalle in Erscheinung, die alle die von der ursprünglichen Trübung durchsetzten Netzhautpartien zum Sitze hatten. Eine besondere Art der Anordnung war dabei nicht erkenntlich. Außerdem fanden sich aber in den vorderen Netzhautschichten auch diese und jene der bei dem zweiten Falle näher zu schildernden Cystchen.

Der zweite Fall, der bei der ersten Untersuchung an der Spaltlampe - also ebenfalls am zweiten Tage nach der Embolie - genau den gleichen Befund wie der erste Fall gezeigt hatte, besserte sich zwar quoad visum und ophthalmoskopischen Augenhintergrundsbefund im Beobachtungsverlanfe von ca. 2 Wochen ganz erheblich, doch blieben die an der Spaltlampe erkenntlichen und oben geschilderten Veränderungen entschieden länger bestehen. Auch hier kam es, wie man etwa aller 3 Tage an der Spaltlampe verfolgen konnte, zu einer fortschreitenden Aufhellung und zu einem allmählichen Verschwinden der gelben Farbe, doeh blieben noch viele "Inseln" von deutlich gelb gefärbter, typisch embolischer Netzhauttrübung bestehen, vor allem im Bereiche der nach der Fovea zu abfallenden Maculapartien kam das zum Ausdruck. Nach ca. 14 Tagen sah man auch in den etwas mehr peripher gelegenen Foveapartien 
hier und da eine gelblich erscheinende Insel, die allem Anscheine nach ebenfalls auf eine der ursprünglichen Netzhauttrübung analoge Veränderung der daselbst gelegenen Netzhautschichten zurückgeführt werden mußte. $\mathrm{Ob}$ dieses Verhalten allerdings für eine auch in der Fovea vorhandene Gelbfärbung sprechen dürfte, wage ich mit Rücksicht auf die an dieser Stelle schon stark durchscheinende tiefere Hintergrundsfarbe nicht zu entscheiden.

Während nun auch hier in dem zweiten Falle sehr frühzeitig, genau wie bei dem ersten Patienten, hier und da auftretende und schon von Wagenmann in einem Embolus gefundene Cholesterineinlagerunge $n$ in der gesamten Maculagegend sichtbar wurden, traten vor allem bei dem zweiten Falle noch weitere interessante Bildungen im Bereiche der Macula sowohl als späterhin auch im Gebiete der Fovea in Erscheinung $\left.{ }^{1}\right),{ }^{2}$ ).

Diese schon oben berührten Bildungen erwiesen sich als allerfeinste in den vorderen und von der Trübung ergriffenen Schichten gelegene Cystchen, die vor allem bei leicht indirektem Lichtauffalle sichtbar waren und das Licht entweder stark reflektierten oder auch als weiße, mehr oder minder oberflächlich gelegene Knöpfchen sich darstellten. Man sah sie allenthalben ziemlich dicht unter der leicht glasig aufgequollenen Limitans liegen und bei Beleuchtungswechsel entweder perlmutterartig schillem oder als die genannten weißlichen Knöpfchen hervortreten. Die Gebilde erschienen von verschiedenster Größe, doch waren die meisten davon bei 70 facher Vergrößerung kaum größer als etwa der Durchmesser einer Präcapillare. Oftmals lagen sie so dicht unter der Limitans und dabei so eng aneinander, daß die Limitans darüber deutlich vorgebuckelt erschien.

Auch in der roten Fovea selber wurden, wie schon angedeutet, nach einigen Tagen die Cystchen sichtbar, namentlich in dem oben geschilderten Stadium des zweiten Falles, woselbst in der Fovea die gelbroten Trübungsinseln auftraten.

Daß es sich bei den besagten, z. T. schillernden Bildungen um Cystchen und keine soliden Bildungen resp. Cholesterinkrystalle handelte, bewies das Spaltlampenbild angesichts der Durchsichtigkeit der Gebilde ad oculos. Außerdem spricht aber dafür der Befund der pathologischen Anatomie, den Nuel nach 6 Wochen, Gowers nach 6 Wochen, Früchte sogar nach 3 Wochen und wiederum einige andere Untersucher nach noch längeren Wochen und Monaten konstatieren konnten.

1) Eine Aussaat von Kalkpartikeln, wie in dem Falle von Coats, kam bei unseren jugendlichen Patienten nicht in Frage.

${ }^{2}$ ) Teils weißlich, teils gelblich gefärbte, fleckenförmige Veränderungen der tiefsten Retinaschichten sowie desPigmentepithels, wie sie z. B. El sch nig [zit. unter (33) Lit.] und Leber beschrieben, sahen wir im Bereiche der Macula oder der Fovea bei unseren Fällen von Embolie nicht, auch nicht nach 3-4 Wochen des Verlaufs. 
Vor allem interessieren uns hier die Beobachtungen von Früchte. Dieser Autor sah nach der angeführten Zeit in seinem Falle stellenweise, besonders nahe der Macula, Vakuolenbildung und leichte Auflockerung der Ganglienzellenschicht. Ferner beobachtete Nuel durch Auftreten zahlreicher Lücken eine netzförmige Beschaffenheit der von der Trübung ergriffenen Netzhautschichten, ein Befund, der mit der sogenannten cystoiden Degeneration der Netzhaut übereinstimmte.

Somit haben wir in unseren Cystehen also aller Wahrscheinlichkeit nach solche postembolische Netzhantvakuolen vor uns, die durch den konsekutiven Gewebszerfall entstanden waren. Da der Befund bei beider. Fallen übereinstimmte, so glauben wir, daß auch weitere Fälle dieser Art die Veränderung zeigen werden, natürlich je nach Alter und Ausbildung des gesamten Prozesses verschieden ausgeprägt.

Als letzte interessante Beobachtung in dem Spaltlampenbilde der gellogefärbten Macula nennen wir bei beiden Fällen im Stadium der Aufhellung hervortretende Faltenbild ungen der Limitans. Diese zeigten sich im direkten Lichte weniger deutlich als im indirekten. Vor allem konzentrisch zur Fovea angeordnet, sah man die das Licht teilweise stark reflektierenden Gebilde als längliche Wälle hier und da in näherer und weiterer Entfernung von der Fovea um dieselbe herumlaufen und über den GefäBchen gelegen ${ }^{1}$ ). Dabei erreichten die Bildungen jedoch niemals eine größere Länge als etwa die Länge des Foveadurchmessers. Mit den Vakuolenbildungen resp. oben geschilderten Cystchen waren die Falten nicht zu verwechseln, davor schützte die unmittelbar unter der Limitansoberfläche befindliche Lage der Gebilde, andererseits aber auch ihre Längenausdehnung sowie thre viel geringere Durchsichtigkeit. Eine eigentliche weißliche Netzhautfalte im Sinne von Har $\mathrm{s}^{2}$ ), welche die Fovea umgab, wurde nicht beobachtet.

Nur im Bereiche der Macula waren die Falten zu sehen. Sie waren offenbar die Folge einer mit dem Verschwinden der weißen Trübung einhergehenden Zusammenziehung der vorderen und infolge der Embolie zunächst übermäßig ausgedehnten Netzhautschichten. Während der ganzen Dauer der Beobachtung blieben sie bei beiden Fällen sichtbar. In den außerhalb der Macula gelegenen Netzhautschichten waren sie nicht mehr zu verfolgen.

Zu der genannten Abbildung haben wir noch des besseren Verständnisses halber hinzuzufügen, daß auf $\mathrm{ihr}$ mindestens 25 Spaltbilder des fovealen, macularen und perimacularen Augenhintergrundsbereiches dargestellt sind. Ein Spaltbild, das auf dem gezeichneten Bilde etwa

1) In unserem 3. Falle sah man auch einige Radiärfältchen dieser Art in der Foveaumgebung.

2) Harms, Archiv f. Ophthalm. 8\%, 2. 1914; auch die kürzlich von Vogt (Lit. 21) beschriebenen echten Retinafältchen kamen hier nicht in Frage. 
die Breite von $8 \mathrm{~mm}$ und eine Höhe von $20 \mathrm{~mm}$ haben würde, ließe also im gelben Maculabezirke einen Streifen der gelben Zone bequem einstellen. Wir haben, um zahlreiche Abbildungen für unsere Darstellung zu ersparen, auf die Zeichnung nur je eines Spaltbildes demgemäß verzichten müssen. Das gilt auch für alle weiteren, nun im folgenden ausgeführten Betrachtungen.

Wir beginnen diese zunächst mit der Untersuchung der in der engeren und weiteren Umgebung des macularen Ringwalles gelegenen Netzhautpartien, wie sie sich in beiden Fällen bei der ersten Untersuchung nach der Embolie darstellten.

Da fiel uns im Spaltlampenbilde vor allem die weiße dichte Trübung der vorderen Netzhautschichten in den Gesichtskreis. Diese Trübung ließ jedoch, was vor allem bei indirekter Beleuchtung und namentlich bei leichtem Oscillierenlassen des Spaltlichtes deutlich war, durchaus nicht etwa eine völlig gleichmäßige Durchsetzung der ergriffenen Netzhautpartien mit der weißlichen ,Trübungsmasse“" exkennen, sondern zeigte sehr deutlich eine Bevorzugung bestimmter Gewebspartien. Und diese Partien waren die zwischen den hiex allenthalben gut hervortretenden und anscheinend kaum veränderten Nervenfaserbündeln sichtbaren "Interfaszikularräume" derselben, wie sie in der zweiten Mitteilung bereits als die Träger des fraglichen retinalen Saftlückensystemes beschrieben wurden. Diese Interfaszikularräume zwischen den Nervenfaserbündeln, die also in unseren Fällen auch im natürlichen Lichte an der Spaltlampe sichtbar waren, sieht man normalerweise, wie im physiologischen Teile ausgeführt, in der weiteren Umgebung der Macula für gewöhnlich nicht mehr. Aber hier bei der embolischen Netzhauttrübung traten sie im Spaltlampenbilde auffallend schön hervor und boten ein interessantes und dankbares Studienobjekt.

Ungefähr in der Hauptverlaufsrichtung der von Vogt makroskopiseh beschriebenen Nervenfaserstrahlung in der weiteren Umgebung der Macula sah man die besagte Netzhauttrübung in länglich parallelen Zügen die Macula umkreisen. Zwischen den ungefähr parallelen Längsbündeln (auf der $\mathrm{Abb}$. nicht gezeichnet) erschienen zahlreiche feinste und nicht sehr regelmäßig angeordnete Querverbindungen, so daß das Ganze den Eindruck eines an der Spaltlampe gerade erkenntlichen feinsten Netzwerkes machte. Bei Oscillationen trat das Netzwerk vor allem auch an der Grenze zu der von der Trübung frei gebliebenen, cilioretinal versorgten und von normaler Farbe erscheinenden Netzhautpartie hervor, welche selbst das uns an der Spaltlampe bekannte Bild darbot und auch, wio gezeichnet, die Nervenfaserstreifung deutlich erkennen ließ.

In den späteren Stadien verlor sich dann die weiße Netzhauttrübung mit der ophthalmoskopischen Aufhellung der ergriffenen Partien, die 
Durchsetzung der Interfaszikularräume, mehr und mehr, ohne jedoch während der Beobachtung ganz zu verschwinden. Dafür traten die weiter unten zu erwähnenden, auch hier beobachteten Gebilde in Erscheinung, wenn auch um vieles spärlicher und undeutlicher.

Von prinzipiellem Interesse exscheint noch die Frage, ob wir, die an der Spaltlampe erkenntliche Bevorzugung der Interfaszikularräume bei der Durchsetzung mit der weißen Trübung diagnostisch genauer deuten können. Die Ähnlichkeit der Durchsetzung mit dem von uns früher geschilderten Bilde der Hornhautsaftlücken beim Hornhautödem läßt uns nämlich vermuten, daß es sich auch hier auf dem Augenhintergrunde neben den Fettkörnchenzellen, den retinalen Zerfallsprodukten und ähnlichem vor allem um ein Ödem ${ }^{1}$ ) handeln müsse, was ja auch durch die oben angeführten anatomischen Befunde bestätigt ist.

Sehr deutlich sah man auch die zur Macula hinstrebenden Rami maculares der sie versorgenden Gefäße. Die arteriellen von diesen ließen z. T. eine völlige Stase der Zirkulation wahrnehmen, wobei die Gefäße selber äußerst dünn und meist kaum sichtbar sich darstellten, während die Venen entschieden erweitert und geschlängelt waren. Die infolge der Stase stilliegende Zirkulation in den erwähnten arteriellen GefäBen zeigte hier und da richtige Geldrollenbildung der feststehenden Blutzellen und vielfache Unterbrechungen der Blutsäule, doch muBte aus technischen Gründen auf eine Darstellung dieser Phänomene verzichtet werden.

Sowohl im frischen Stadium wie auch noch nach 2 Wochen konnte man diese and jene der macularen präcapillaren Ästchen über den macularen Ringwall hinweg bis etwa zum Beginne des mittleren Drittels des Foveaabfalles verfolgen. Namentlich in dem zuletzt geschilderten späteren Stadium war das besonders deutlich, während kurz nach der Embolie diese Ästchen wie auch zum Teile die in der weiteren Umgebung der Macula zu dieser hinverlaufenden Rami maculares hier und da mehr oder weniger von der weißen Retinatrübung verdeckt erschienen. Am inneren Rande des Ringwalles erfolgte namentlich im späteren Stadium eine ziemlich stark ausgesprochene Abknickung der Gefäßchen. Auch hier waren sie im ersten Stadium meist verdeckt, späterhin dagegen meist frei und ziemlich dicht anter der Oberfläche verlaufend. Hier kam auch ein deutliches Anastomosennetz der Rami in ihren letzten noch erkennbaren Ausläufern zur Wahrnehmung, offenbar handelte es sich hier um das sonst und unter physiologischen Umständen nicht sichtbare perifoveale resp. maculare, capillare Anastomosennetz.

In der weiteren Umgebung der gelben Macula, speziell des Ringwalles, fanden sich einige kleinere und größere Blutungen in der weiß

1) Bei der Besprechung der übrigen Formen des retinalen Ödems werden wir noch genauer und auch differentialdiagnostisch darauf zurückzukommen haben. 
getrübten Netzhaut, und zwar meist dicht unter der Limitans. Die eine größere Blutung dieser Art ist gezeichnet. Man konnte das Blut in Gestalt eines ein paramaculares Gefä $B$ - anscheinend eine feinste Vene - umscheidenden Konglomerates von roten Blutzellen und späterhin auch von in körniger Umwandlung begriffenem Blutfarbstoff deutlich erkennen. Die Limitans darüber erschien mehr oder minder abgehoben. In der Übergangszone zur weiß getrübten Nachbarsehaft verlor sich das Blutmaterial in allerfeinsten Streifen leichthin unter der Netzhautoberfläche und schien vor allem den Interfaszikularräumen zu folgen. Auch die übrigen feineren Blutungen boten das gleiche Bild.

In der weiteren Umgebung der Macula zeigten sich nur noch ganz vereinzelte Cysten, auch die Cholesterinkryställlchen waren bedeutend weniger zu sehen. Im späteren Stadium sah man aber des weiteren noch hier und da etwa radiär zur Papille verlaufende und über den Gefäßen gelegene Limitansfalten, welche das Licht stark reflektierten und an die von $\nabla$ ogt im rotfreien Lichte gesehenen Bilder erinnerten. Daß es sich speziell in unseren Fällen um solche Faltungen der Limitans und unter keinen Umständen um etwaige Fältelungen der Hyaloidea handeln konnte, wie Vogt glaubte diese Bildungen meistens deuten zu müssen, bewies das Spaltlampenbild mit unwiderlegbarer Klarheit. Wir werden in den kommenden Mitteilungen noch öfters von diesen Limitansfalten zu sprechen haben ${ }^{1}$ ). In unseren. Fällen war ja ihre Entstehung aus der sich wieder zusammenziehenden Netzhaut nach ihrer Ausdehnung durch die embolische Trübung ohne weiteres verständlich, doch wurde eine Teilung, ein Zackigwerden oder eine doppelte Kontur der Falten resp. der durch sie gesetzten Reflexe nicht wahrgenommen ${ }^{1}$ ).

Was nun speziell den von der Trübung ophthalmoskopiseh nicht ergriffenen Bezirk im einzelnen betraf, so zeigte er, wie auch auf der Abbildung angedeutet, die Nervenfaserstreifung auch im rothaltigen Lichte sehr deutlich. Vor allem an der Grenze zur weißlich getrübten Nachbarschaft ließ sich jedoch dèr Ausbreitungsmodus der weißen Embolietrübung der Netzhaut sehr deutlich erkennen und zeigte in seiner Bevorzugung des interfaszikularen Saftlückennetzwerkes die oben erörterten Eigentümliehkeiten.

Auch bei unserem zweiten Falle wurde die auf der Abbildung dargestellte weißliche Brücke zur gelben Macula zwar allmählich schmaler und schmaler, doch auch im zuletzt beobachteten Wiederaufhellungsstadium der Erkrankung blieb immer noch eine ausgedehnte, nur weiß und nicht gelb gefärbte Zone zwischen dem cilioretinalen Bezirke und dem Beginne des gelben maculären Ringwalles bestehen. Die Breite

1) Deshalb möchte ich auch auf die Frage der pathologischen Netzhautreflexe heute nicht gesondert eingehen, sondern den Gegenstand für die Retinitis noch aufsparen. 
dieses Grenzstreifens bemaß sich ungefähr zu dem Durchmesser der gesamten Gelbfärbung.

Damit kommen wir zu den im Spaltlampenbilde sichtbar gewordenen Veränderungen der Papille.

Ophthalmoskopisch zeigte sich uns der Pfropf am zweiten Tage nach der Embolie in beiden Fällen auf der Papille da, wo er wegen der ungünstigen Passage durch die Lamina cribrosa am häufigsten angetroffen wird, nämlich an der Zweiteilung der Zentralarterie unmittelbar nach ihrem Durehtritte durch die Lamina. Hier sah man sowohl im umgekehrten wie vor allem auch im aufrechten Bilde den Pfropf als dunkle Masse unmittelbar unter der Zweiteilung unbeweglich in dem Hauptstamme der Zentralarterie sitzen, während die peripher davon befindlichen arteriellen Zweige völlig kollabiert und blutleer sich darstellten resp. - im aufrechten Bilde an einigen größeren Ästen der oberen und unteren Verzweigungen der. Temporalarterien gerade noch eben sichtbar - eine Stase ihres Blutinhaltes mit ein- oder mehrfacher Unterbrechung der Blutsäule erkennen ließen. Die Venen waren im großen und ganzen kaum oder nicht wesentlich verändert. Im frischen Stadium war auch die Papillenfarbe wenig alteriert, sie zeigte außer einer geringen Anämie eine nur geringfügige ödematöse Aufquellung mit leichter Verwaschung der Grenzęn. Eine Faltenbildung der Limitans resp. Radiärreflexe im Papillengebiete ${ }^{1}$ ) wurden nicht beobachtet.

An der Nernstspaltlampe wạ dieses frische Stadium dadurch charakterisiert, daß das ganze Gewebe ausgesprochen aufgequollen und undurchsichtiger als normal sich darstellte. Die perivasculären Lymphscheiden der Papillengefäße wie auch diese und jene der solitären Lymphgefäße am Papillenrande schienen vielleicht etwas mehr gefüllt und deutlicher zu sein als sonst, was offenbar auf eine Verlangsamung des gesamten Säftestromes in dieser Gegend hindeutete.

Ein schönes Bild bot der die Zentralarterie obliterierende Pfropf selbst. Man sah ihn in dem leicht aufgequollenen Hauptstamme der Zentralarterie undeutlich sitzen und daselbst durch die hier noch ziemlich starke Wandung der Arterie undeutlich hindurchschimmern. Er erschien von trüb grauroter Farbe, zeigte aber wegen der darum befindlichen Arterienwandungen keine feiner differenzierbaren histologischen Einzelheiten. Die perivasculäre Lymphscheide der Zentralarterie erschien im Bereiche des Pfropfes getrübt, exsudativähnlich aufgequollen und verbreitert, was späterhin wieder völlig verschwand.

Die sämtlichen mit der Zentralarterie zusammenhängenden Papillengefäße wie auch ihre Fortsetzungen in die übrige Netzhaut hinein waren kollabiert, ziemlich durchsichtig und verdünnt. Später, als durch Wiederherstellung der Zirkulation infolge spontaner Lösung und Zerteilung

1) Vgl. bei Vogt; Klin. Monatsschr. f. Augenheilk. 58, 590. 1917. 
des Pfropfes die Zirkulation, wenn auch nur mangelhaft, so sich aber doch wiederherstellte, nahmen sie wieder in dem getrübten Netzhautgewebe da, wo sie sichtbar und durch die weiße Netzhauttrübung nicht verdeckt oder verschleiert waren, ihr ungefähres sonstiges Aussehen wieder an, abgesehen natürlich von einer ausgesprochenen Kaliberverminderung.

Ein besonderes Verhalten der perivasculären Lymphscheiden war an den verengten arteriellen Asten der Zentralarterie in den der Spaltlampenuntersuchung zugänglichen Netzhautpartien nicht wahrzunehmen, auch ließen die Venen keine auffäligeren histologisehen Veränderungen erkennen.

Im späteren Verlaufe wurde bei beiden Fällen die Papille bedentend weißlicher, ja, fast atrophisch, trotz des erst relativ kurze Zeit von 2-3 Wochen währenden Verlaufes der embolischen Veränderungen und teilweiser Wiederherstellung der Zirkulation, wobei der Pfropf sich an der Spaltlampe eines Tages plötzlich verschwunden zeigte. Das erwähnte Ödem der Papillen ging zum größten Teile zurück, während die Gefäßè sowie ihre Lymphscheiden ihr altes Aussehen wieder annahmen. Dabei ließen sich auch in der näheren Umgebung des Opticus in beiden Fällen hier und da bei noch weißlich getrübtem Netzhautgewebe in dessen Innerem vereinzelte glitzernde Kryställehen nachweisen, die ihrem im Spaltlampenbilde zu beobachtenden Verhalten nach auch hier als Cholesterin angesprochen werden muBten.

Aus dem Umstande, daß wir bei den beiden beobachteten Fällen von Embolie der Zentralarterie die geschilderten Bilder vermittels unserer neuen Apparatur auch an den extramacularen Teilen des Augenhintergrundes nachweisen konnten, glauben wir den SchluB ziehen zu dürfen, daß das geschilderte Spaltlampenbild für die embolischen Augenhintergrundsveränderungen typisch war. Selbstredend werden wir anch fernerhin mit unserex Apparatur weiteres Material dieser Art aufmerksam zu verfolgen und mit den uns bisher über das Krankheitsbild der Embolie aus der pathologischen Anatomic bekannt gewordenen Tatsachenmateriale in engste Beziehung zu setzen haben.

Eine zusammenfassendere Übersicht über das Gesagte sowie vor allem auch über die erreichten neuen Feststellungen mag zum Schlusse unseren Untersuchungen einen bestimmteren Ausdruck verleihen. So legt uns an der Hand unserer Untersuchungen des lebenden Augenhintergrundes im fokalen Lichte der Gullstrandschen Nernstspaltlampe das Augenhintergrundsbild bei der Embolie der Zentralarterie folgende Sehlüs se nahe:

1. Die Spaltlampenuntersuchung des lebenden Augenhintergrundes bei der Embolie der Zentralarterie beweist uns die Tatsache, daß zum mindesten die perifovealen Maculapartien gelb gefärbt sein müssen 
und damit die Behauptung Vogts betreffs der intravitalen Gelbfärbung der Macula richtig ist.

2. In den späteren Stadien der embolischen Netzhautveränderungen scheint auch eine gewisse Beteiligung der tieferen Netzhautpartien stattfinden zu können, was aus den im Gebiete der eigentlichen Fovea centralis zu beobachtenden Cystenbildungen hervorgeht und anatomisch vor allem schon von Elschnig gezeigt wurde.

3. Aus der dem Spaltlampenbilde nach fast ausschlieBlichen Beteiligung des interfaszikularen fraglichen Saftückensystemes bei der weißen Trübung der lebenden Netzhaut im Gefolge der Embolie der Zentralarterie darf geschlossen werden, daß hier neben den anatomisch festgestellten zelligen und degenerativen Veränderungen dieses Gewebes tatsächlich auch ein Odem besteht und somit die gleichen anatomischen Feststellungen Lebers, Ruberts, Siegrists u. a. auch für das lebende Auge Gültigkeit besitzen.

4. Bei der nach der Embolie der Zentralarterie auftretenden Netzhautveränderung lehrt uns die. Gullstra n d sche Nernstspaltlampe, daß die von Vogt für Fältelungen der Hyaloidea gehaltenen und über den Gefäßen gelegenen Radiärreflexe auf dem pathologisch veränderten lebenden Augenhintergrunde wenigstens für die postembolische Netzhauttrübung in echten Faltenbildungen der Limitans interna zu erklären sind.

\section{Literatur.}

1. Coats, zit. nach (14).

2. Dimmer, F., Die Nacula lut. der menschlichen Netzhaut usw. Archiv f. Ophthalmol. 65.

3. Elschnig, A., Über die Emb. d. Art. centr. retin. Archiv f. Augenheilk. 24. 1892 .

4. Früchte, Zur Frage d. Emb. d. Art. centr. ret. Klin. Monatsschr. f. Augenheilk. 46, 1. 1908.

5. Gowers, A case of simultaneous \& c. Lancet. 4. XII. 1875.

6. Gullstrand, A., Die Farb. d. Mac. centr. ret. Arohivf. Ophthalmol. 62. 1905.

i. - Zur Maenlafrage. Archiv f. Ophthalmol. 66. 1907.

8. - Klin. Monatsschr. f. Augenheilk. März 1918.

9. Haab, Augenspiegelstudien. Archiv f. Augenheilk. 81. 1916.

10. v. d. Hoeve, J., Die Farbe der Mlac. lut. Archiv f. Opththalm. 80. 1911.

11. Koep pe, L., Klin. Beob. m. d. Nernstsp. Mitteil. XIV. Archiv f. Ophthalmol. 97. 1918.

12. - Die Mikroskopie d. leb. Augenhintergr. usw. II. Archiv f. Ophthalmol. 97. 1918.

13. - Die Lösung der Streitfrage usw. Münch. med. Wochenschr. 43. 1918.

14. Leber, Th., Die Krankheiten d. Netzhaut. Handb. v. Graef.-Säm. VIII. A. 1915.

15. Lottrup-Andersen. Klin. Mon. f. Augenheilk. 51, 1. 1913.

16. Nuel, Altérat. de la macul. C. Embol. de l'art. centr. etc. Archiv d'Ophthalmol. 16. 1896. 
78 L. Koeppe: Die Mikroskopie des lebenden Augenhintergrundes usw.

17. Rubert, Über d. Emb. d. Art. centr. ret. Klin. Monatsschr. f. Augenheilk. 49, 2. 1911 .

18. Siegrist, Die Ge. d. Lig. usw. Archiv f. Ophathalmol. 50. 1900.

19. Vogt, A., Herstellung eines gelbbl. Lichtfilt. usw. Archiv f. Ophthalmol. 84. 1913 .

20. - Heidelberger Bericht 1913.

21. - Klin. Monatsschr. f. Augenheilk. 58, 399ff, ferner 587ff. 1917.

22. - Klin. Monatsehr. f. Augenheilk. 60, $449 \mathrm{ff}$. und 61, $381 \mathrm{ff} .1918$.

23. Wagenmann, Ein Beitrag z. Kenntnis der path. Anat. d. Emb. d. Zentralart. Archiv f. Ophthalmol. 40, 3. 1894.

24. Wolff, H., Die reflexl. Ophthalmomikroskopie. Zeitschr. f. Augenheilk. 28. 1913. 
v. Graefes Archiv Bd. 99.

Tafel II.

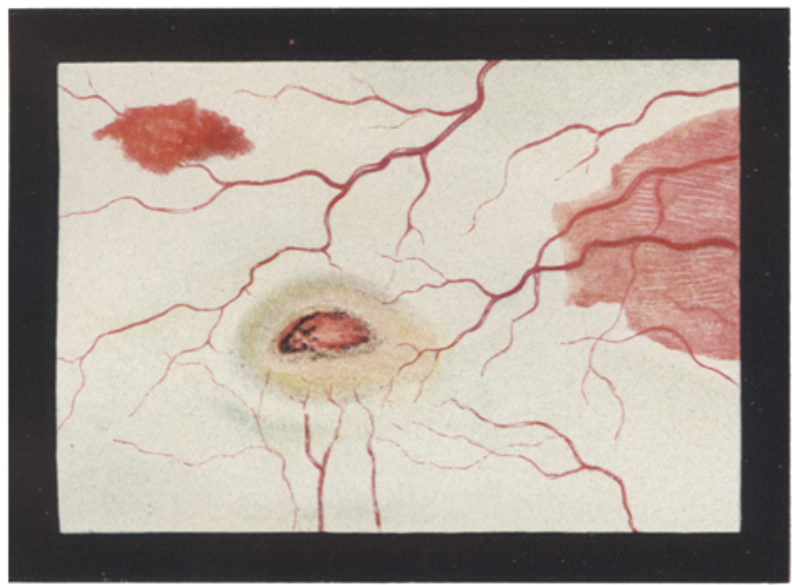

